\title{
COMPARISON OF THE ABUNDANCES IN THE STARS AND IN THE HII REGIONS OF THE MAGELLANIC CLOUDS
}

\author{
M. SPITE, F. SPITE \\ Observatoire de Paris-Meudon \\ 92195 Meudon Cedex \\ France
}

\begin{abstract}
Both HII regions and F supergiants are young objects, and are, therefore, supposed to have the same abundances for all elements, especially for $\mathrm{C}, \mathrm{O}$ and $\mathrm{S}$, which offer a good opportunity to check the abundance determinations made by two different techniques. For oxygen and sulfur the agreement is good but the discrepancy of the carbon abundance cannot be easily explained.
\end{abstract}

\section{Introduction}

The determination of the metallicity of stars in the Magellanic Clouds is important for our understanding of their chemical evolution which, in turn, is a useful parallel to the evolution of the Galaxy. The determination of the abundance of the light elements like $\mathrm{C}$ and $\mathrm{O}$ are of particular interest since, after $\mathrm{H}$ and $\mathrm{He}$, they are the most abundant in the Universe. Unfortunately, the abundance of carbon as deduced from the HII regions (Dufour 1984) is different from that as deduced from the young stars (Spite et al. 1989a, 1989b, Russell and Bessell 1989). The aim of this work is a systematic comparison of the abundances of the elements which can be measured both in the HII regions and in the F field supergiant stars.

\section{Observations}

The abundance of the light elements carbon, oxygen and sulfur has been measured in four stars of the Magellanic Clouds: three in the SMC and one in the LMC. The analysis of the stars in the SMC was made on the basis of data (Spite et al. 1989) collected at the European Southern Observatory with the $3.6 \mathrm{~m}$ telescope and the CASPEC spectrograph; the resolving power is 20000 and the signal-to-noise ratio about 100 . The receptor was a CCD RCA chip $(512 * 320$ pixels with a $30 \mu \mathrm{m}$ size). Similar spectra have also been obtained for stars in the LMC. Complementary spectra of field stars AZ369 in the SMC, G258 in the LMC, and of the galactic F supergiants $\alpha$ Car and $\alpha$ Lep (reference stars) have been obtained with the ESO-CES spectrograph with a resolving power of 40000 centred on the carbon line $(538 \mathrm{~nm})$ and the forbidden oxygen line $(630 \mathrm{~nm})$.

\section{Result of analysis, conclusion}

The elemental abundances were determined by fitting computed spectra to the observed ones (Figure 1). 


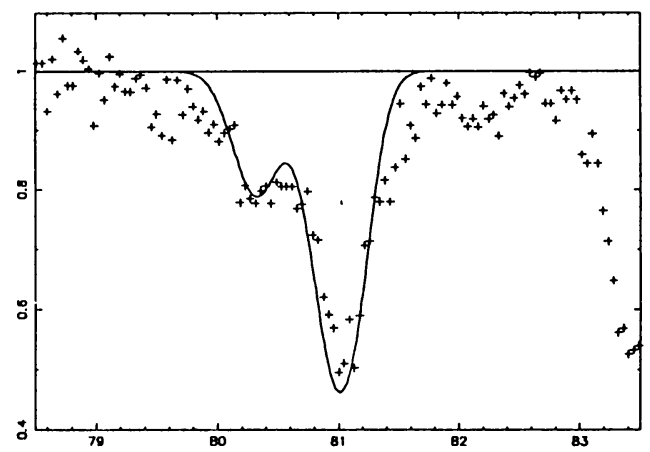

Figure 1. Synthetic (full line) and observed spectrum (crosses) of the field F supergiant of the LMC: G258 in the region of the carbon line at $538.032 \mathrm{~nm}$. The computations have been made with $\log \mathrm{N}_{\mathrm{C}}=$ 8.11 (for $\log \mathrm{N}_{\mathrm{H}}=12$ ).

The Kurucz (1979) models were used to represent the atmosphere of the stars; the $g f$ values for the different lines are given in Spite et al. (1989b) and Spite and Spite (1990). Since a systematic error can affect the abundance determination in supergiant stars and in HII regions, it is important to compare the abundances obtained in the Magellanic objects with the abundances of similar objects in our Galaxy. The results for the Small and the Large Magellanic Clouds are presented in the next table.

\begin{tabular}{|c|c|c|c|}
\hline & Carbon & Oxygen & Sulfur \\
\hline $\begin{array}{l}\text { GALAXY } \\
\text { Sun } \\
\text { Mean Galactic F supergiant } \\
\text { Galactic Hil regions }\end{array}$ & $\begin{array}{l}\log N \\
8.56 \\
8.36 \\
8.46\end{array}$ & $\begin{array}{l}\log N \\
8.93 \\
8.73 \\
8.70\end{array}$ & $\begin{array}{l}\log N \\
7.21 \\
7.26 \\
7.06\end{array}$ \\
\hline $\begin{array}{c}\text { SMC } \\
\text { AZ140/gal. F supergiant } \\
\text { AZ197 / gal. F supergiant } \\
\text { AZ369 / gal. F supergiant }\end{array}$ & $\begin{array}{c}\Delta \log N \\
-0.70 \\
-0.75 \\
-0.60\end{array}$ & $\begin{array}{c}\Delta \log N \\
-0.72 \\
-0.57 \\
-0.62\end{array}$ & $\begin{array}{c}\Delta \log N \\
-0.64 \\
-0.43 \\
-0.64\end{array}$ \\
\hline $\begin{array}{c}\text { Mean star Bessell-Russell/ } \\
\text { gal. F supergiant }\end{array}$ & -0.77 & $* * *$ & $* * *$ \\
\hline $\begin{array}{l}\text { General stellar mean } \\
\text { SMC HII regions / Gal. HII reglons }\end{array}$ & $\begin{array}{l}-\overline{0.76} \\
-1.30\end{array}$ & $\begin{array}{l}-\overline{0} . \overline{64} \\
-0.68\end{array}$ & $\begin{array}{l}-\overline{0} . \overline{5} \overline{7} \\
-0.57\end{array}$ \\
\hline $\begin{array}{c}\text { LMC } \\
\text { G258 / gal. F supergiant }\end{array}$ & $\begin{array}{l}\Delta \log N \\
-0.25\end{array}$ & $\begin{array}{c}\Delta \log _{-0.30} N \\
\end{array}$ & $\begin{array}{c}\Delta \log N \\
-0.26\end{array}$ \\
\hline $\begin{array}{c}\text { Mean star Bessell-Russell } \\
\text { gal. F supergiant }\end{array}$ & -0.29 & $* * *$ & 0.44 \\
\hline $\begin{array}{l}\text { General stellar mean } \\
\text { SMC HII regions / Gal. HII regions }\end{array}$ & $\begin{array}{l}-0.27 \\
-0.75\end{array}$ & $\begin{array}{l}-0.30 \\
-0.44\end{array}$ & $\begin{array}{l}-\overline{0.35} \\
-0.21\end{array}$ \\
\hline
\end{tabular}

It can be seen that if carbon seems to be, surprisingly, three times more deficient in the HII regions than in the field stars, there is good agreement for both oxygen and sulfur in the Small and in the Large Magellanic Cloud.

\section{References}

Dufour, R.J. (1984), IAU Symp. 108 Structure and Evolution of the Magelllanic Clouds, S. van den Bergh and K.S. de Boer (eds.) (Reidel: Dordrecht) p. 353.

Kurucz, R.L. (1979), Astrophys. J. Suppl. Ser. 40, 1.

Russell, S.C., Bessell, M.S. (1989), Astrophys. J. Suppl. Ser. 70, 865.

Spite, F., Spite, M., François, P. (1989a), Astron. Astrophys. 210, 25.

Spite, M., Barbuy, B., Spite, F. (1989b), Astron. Astrophys. 222, 35.

Spite, M., Spite, F. (1990), Astron. Astrophys. in press. 